\title{
Sensitisation to Hodgkin's disease spleen tissue in patients with malignant lymphoma: follow-up study
}

\author{
B W HANCOCK, LESLEY BRUCE, JOHN RICHMOND
}

British Medical fournal, 1976, 2, 351-352

\section{Summary}

The leucocyte migration responses of patients with malignant lymphoma to an unidentified factor in Hodgkin's spleen tissue were serially studied and related to clinical progress. Initial sensitisation responses did not correlate with presenting histological or clinical status or with subsequent clinical progress. Enhancement of responses after treatment, however, was associated with good clinical progress. In patients who relapsed, sensitisation to spleen factor diminished, whereas responses were preserved at one year in those in maintained remission. Sensitisation to the splenic factor may be a useful index of response to treatment in patients with malignant lymphoma; diminishing sensitisation may indicate relapse.

\section{Introduction}

We have shown, using the leucocyte migration inhibition technique, sensitisation to splenic tissue taken from a patient with Hodgkin's disease in about half of a group of patients with malignant lymphoma. ${ }^{1}$ In a follow-up study we assessed the relation of the leucocyte migration response in such patients to their subsequent clinical progress after treatment.

\section{Patients and methods}

Seventy-seven patients with malignant lymphoma were assessed at presentation and after completing radical radiotherapy (48 patients) or before the fourth course of intensive quadruple cytotoxic chemotherapy (29 patients).

These patients were observed over the next three months to establish their initial response to treatment. Fifty-three patients achieved complete remission (good response); 12 showed incomplete regression of clinical and investigative indices of disease activity (partial response), and 12 showed no response to treatment (poor response).

Eight of the patients with a poor response and two of those with a partial response died within six months of their second assessment without responding to further treatment. The remaining patients were followed for nine to 12 months after their second assessment. Nine patients relapsed after apparently successful treatment; these patients were assessed during relapse. Twenty-four patients were in complete remission for 12 months after the start of treatment and were reassessed.

The leucocyte migration technique used was that described by S $\phi$ berg and Bendixen ${ }^{2}$ with minor modifications. ${ }^{1}$ Separated washed peripheral blood lymphocytes were allowed to migrate from microcapillary tubes in wells containing (a) homogenised necropsy splenic tissue from a patient with Hodgkin's disease at a concentration of $10 \mathrm{mg} / \mathrm{l}$ in nutrient medium and $(b)$ control medium.

Migration areas with splenic extract in each experiment were statistically compared with control migration areas (Student's $t$ test),

Academic Division of Medicine, University of Sheffield, Royal Hospital, Sheffield S1 3SR

B W HANCOCK, MB, MRCP, lecturer

LESLEY BRUCE, HNC, medical laboratory scientist

JOHN RICHMOND, MD, FRCP, professor and significant migration inhibition was said to have occurred if $\mathrm{P}<0.05$.

A migration index was also obtained by dividing the area of migration with splenic factor by the area of migration in the control medium.

To assess reproducibility of the technique six patients (four with significant migration inhibition and two with no migration inhibition) were reassessed two (two patients) or three (four patients) times over 10 days during which their disease activity remained substantially unchanged.

\section{Results}

In those patients tested sequentially during a period of unchanged disease activity the migration indices did not vary significantly; the variation from the first result was less than $5 \%$ in all patients.

There was no correlation between presenting histological and staging status and leucocyte migration indices, or between initial migration indices and subsequent response to treatment.

After treatment there was a significant enhancement $(P<0.001)$ in migration inhibition in patients responding to treatment (see figure). Thirty-four of these 53 patients $(64 \%)$ showed enhanced migration inhibition, and $36(68 \%)$ showed significant inhibition with splenic factor after treatment compared with $25(47 \%)$ at presentation.

Enhanced migration inhibition was also seen in the 12 who responded partially to treatment; this change was not, however, significant. Five of these $12(42 \%)$ patients improved their responses; six $(50 \%)$ showed significant migration inhibition after treatment, the same number as before treatment.

Diminished (non-significant) responsiveness was seen in those patients not showing clinical improvement after treatment. Only two of the 12 patients $(17 \%$ ) showed improved responses, and only two patients $(17 \%)$ showed significant inhibition of migration (compared with six reacting before treatment).

The mean migration indices ( $t$ SE of mean) after treatment in the good and partial response groups $(0.77 \pm 0.01$ and $0.80 \pm 0.03$ respectively) were significantly better $(P<0.001$ and $P<0.02$ respectively) than that in the poor response group $(0.90 \pm 0.03)$.

The values in patients who relapsed after treatment showed significantly $(P=0.05)$ diminished responsiveness (mean $0.94 \pm 0.03$ ) compared with post-treatment values (mean $0.84+0.04$ ). They showed some diminished responsiveness when compared with pretreatment

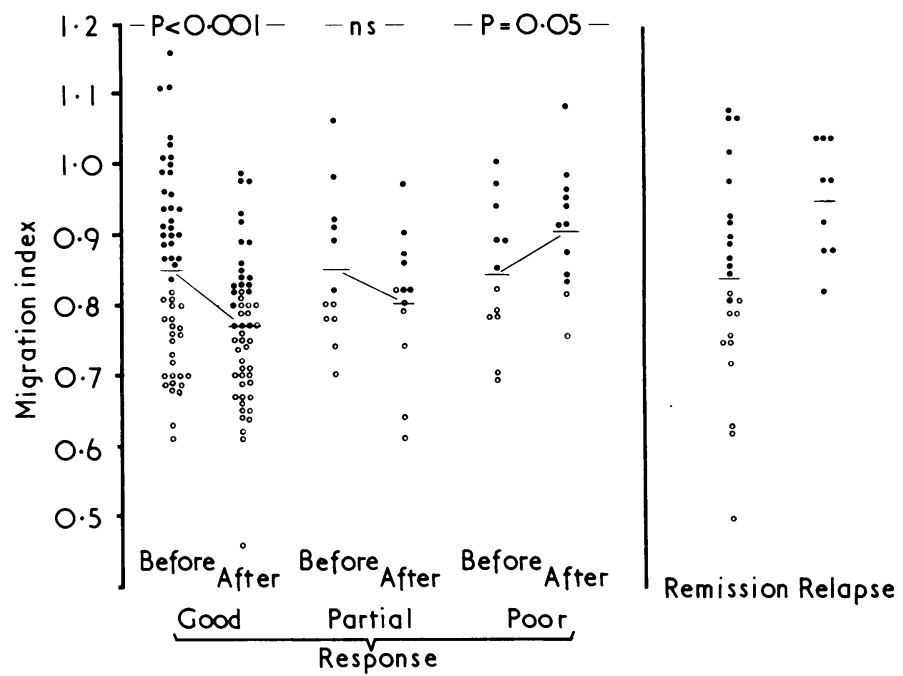

FIG 1-Migration indices with necropsy spleen extract in patients before and after treatment and in relapse and remission.

$\mathrm{O}=$ Significant $(\mathbf{P}<0.05)$ inhibition of leucocyte migration. 
responses (mean $0.90 \pm 0.03$ ), but this was not significant. None of the patients in relapse showed significant migration inhibition with the splenic factor (see figure).

Those patients in remission 12 months after starting treatment showed no significant differences between remission migration response $(0.83 \pm 0.03)$ and pretreatment and post-treatment responses $(0.87 \pm 0.03$ and $0.81 \pm 0.02$ respectively). Eleven of the 24 patients $\left(46^{\circ}{ }_{0}\right)$ in remission showed significant migration inhibition compared with $12\left(50_{0}^{\circ}\right)$ at presentation.

The responses of patients in remission were significantly better $(\mathbf{P}<0.05)$ than the responses of patients in relapse.

\section{Discussion}

Possible antigens have been shownin non-cultured and cultured Hodgkin's disease tissues. ${ }^{3-7}$ Using the leucocyte migration technique, sensitisation to a factor in spleen obtained at necropsy from a patient with Hodgkin's disease was observed in about half the patients with malignant lymphoma tested. ${ }^{1}$ Sensitisation was less common in patients with other varieties of malignant disease $(10 \%)$, and rare in other inpatients and normal controls.

Patients with a positive immunological response to their tumour might be expected to have a better prognosis than those without such immunity. In our patients initial sensitisation to the splenic factor did not correlate with subsequent progress, nor was there any relation between presenting histological and clinical status and initial sensitisation responses. Nevertheless, when migration inhibition responses were retested during or after treatment and the patient's subsequent progress observed it was evident that enhancement of leucocyte migration inhibition at this stage was associated with good clinical progress. In subsequent assessment deterioration of response was found $m$ in patients relapsing whereas those in maintained remission? preserved their sensitisation to the splenic factor.

These findings suggest that sensitisation to the splenic factor, as yet unidentified, may be a useful index of response to treatment $\Rightarrow$ in patients with malignant lymphoma; deterioration of sensiti- $\stackrel{\vec{\rho}}{\rightarrow}$ sation may portend relapse of disease.

We are grateful to the consultant staff of Weston Park Hospital for access to patients under their care and to the Cancer Research $\mathbb{\Phi}$ Campaign (Yorkshire Branch) for financial assistance.

\section{References}

${ }^{1}$ Hancock, B W, Bruce, L, and Richmond, J, British Medical fournal, 1976, $1,556$.

2 S $\phi$ berg, M, and Bendixen, G, Acta Medica Scandinavica, 1967, 181, 247.

${ }^{3}$ Order, S E, Porter, M, and Hellman, S, New England fournal of Medicine, N 1971, 285, 471.

${ }^{4}$ Eshhar, Z, Order, S E, and Katz, D H, Proceedings of the National Academy of Science of the United States of America, 1974, 71, 3956.

${ }^{5}$ Long, J C, et al, Proceedings of the National Academy of Science of the $W^{W}$ United States of America, 1973, 70, 1540.

${ }^{6}$ Long, J C, Aisenberg, A C, and Zamecnik, P C, Proceedings of the National Academy of Science of the United States of America, 1974, 71, 2285.

7 Long, J C, Aisenberg, A C, and Zamecnik, P C, Proceedings of the National Academy of Science of the United States of America, 1974, 71, 2605.

\section{Closed mitral valvotomy}

\section{KENNETH FRASER, MURDO A TURNER, BRIAN A SUGDEN}

British Medical fournal, 1976, 2, 352-353

\section{Summary}

The results of closed mitral valvotomy operations in 359 patients operated on from August 1957 to October 1974 were assessed at July 1975 . About $60^{\circ}{ }_{0}$ of the patients were in good health. These results suggest that there is still a place for closed mitral valvotomy in carefully selected cases.

\section{Introduction}

During the past 18 months the results of open mitral valvotomy were compared with the results of the closed operative procedure. ${ }^{12}$ Cogent arguments exist both for and against the closed technique, which is accepted by most surgeons as an acceptably safe means of relieving critical stenosis in suitable cases. The long-term results of both closed and open methods should ideally be concurrently compared for a true comparison to be drawn, but the reduced number of cases of mitral stenosis presenting at present would probably not provide reliable results in such a trial.

Department of Surgery, Western Infirmary, Glasgow G11 6NT KENNETH FRASER, CHM, FRCS ED, senior lecturer MURDO A TURNER, MB, FRCS, consultant cardiothoracic surgeon BRIAN A SUGDEN, MB, FRCS GLAS, surgical registrar
We therefore decided that information of some value might accrue from a further review of cases published in the British Medical fournal with, in addition, the results in patients operated on since these papers were published. ${ }^{3-5}$ This further review includes all patients with mitral stenosis who had a primary. closed valvotomy before October 1974.

\section{Patients and methods}

The results of closed mitral valvotomy operations performed by three surgeons in the Western Infirmary, Glasgow, in 1957-74ㄱ. were reviewed. The operation was carried out using the Tubbs mitraln valve dilator passed through the transventricular route. ${ }^{6}$ Of the $359 \mathrm{~N}$ patients who underwent this procedure the surviving 287 were $\omega$ available for assessment. Most were reviewed as outpatients and when this was not possible because of length of distance to travel or disability, information was obtained from the patient, his doctor, or both. Fourteen $(4 \%)$ of the group were not available for study. Three? patients were not traced after discharge from hospital and another 110 were lost at later stages of follow-up, some being under surveillance for up to nine years before they left the district. A few patients were traced through the Executive Council list owing to local population $\mathbb{\Phi}$ movement within the area.

\section{Results}

The 359 patients were divided into three groups: group 1 underwent operation in 1957-62, group 2 in 1963-8, and group 3 in 1969-74. Table I shows the decline in the number of operations performed more recently, thought to be due partly to the decline in the number of patients presenting with rheumatic heart disease but also to the increased availability of open heart surgery. 